\title{
ON CERTAIN SERIES INVOLVING RECIPROCALS OF BINOMIAL COEFFICIENTS
}

\section{NECDET BATIR}

Abstract. We evaluate the following family of series involving reciprocals of binomial coefficients in terms of elementary functions for $m=3,4$.

$$
\sum_{k=0}^{\infty} \frac{x^{k}}{(m k+1)\left(\begin{array}{c}
m k \\
k
\end{array}\right)} .
$$

Mathematics subject classification (2010): Primary 11Y60; Secondary 40A05..

Keywords and phrases: binomial sum, series, binomial coefficients, catalan constant, Apéry constant, integral representations.

\section{REFERENCES}

[1] R. APERY, Irrationalite de $\zeta(2)$ et $\zeta(3)$, Journes Arithmetiques de Luminity, Asterisque, 61 (1979), $11-13$.

[2] N. BATIR, Integral representations of some series involving $\left(\begin{array}{c}2 k \\ k\end{array}\right)^{-1} k^{-n}$ and some related series, Appl. Math. Comput., 147 (2004), 645-667.

[3] N. BAtIR, On the series $\sum_{k=1}^{\infty} \frac{x^{k}}{k^{n}\left(\begin{array}{c}3 k \\ k\end{array}\right)}$, Proc. Indian Acad. Sci. Mathematical Sciences, 115, 4 (2005), $371-381$.

[4] J. Borwein, R. Girgensohn, Evaluations of binomial series, Aequationes Math., 70 (2005), 2536.

[5] A. I. Davydychev and M.Yu. Kalmykov, Massive Feynmann diagrams and inverse binomial sums, Nuclear Physics B, 699, (1-2) (2004), 3-64.

[6] A. Sofo, On sums of binomial coefficients, Proyecciones, 28, 1 (2009), 35-45.

[7] A. Sofo, Some properties of reciprocals of double binomial coefficients, Tamsui Oxf. Math. Sci, 25, 2 (2009), 141-151.

[8] A. Sofo, Harmonic numbers and double binomial coefficients, Integr. Transf. and Spec. F., 20, 11 (2009), 847-857.

[9] A. SoFo, Some estimates and identities involving reciprocals of binomial coefficients, The $50^{\text {th }}$ Annual Meeting of the Australian Math Soc., 25-29 September, 2006, Macquarie University.

[10] A. SoFo, Integrals and polygamma function representations for binomial sums, J. Integer Sequences, 13 (2010), Article 10.2.8.

[11] R. SPRUGNOLI, Sums of reciprocals of certain binomial coefficients, Electronic J. Combin., Number Theory, 6 (2006), 1-17.

[12] B. Sury, Tianming Wang And Feng-Zhen Zhao, Some identities involving reciprocals of Binomial coefficients, J. Integer Sequences, 7 (2004), Article 04.2.8

[13] Jin-Hua YAng, Feng-Zhen ZhaO, Certain sums involving inverses of binomial coefficients and some integrals, J. Integer Sequences, 10 (2007), Article 07.8.7.

[14] Z. NAN-YUE AND K.S. Williams, Values of the Riemann zeta function and integrals involving $\log \left(\sinh \frac{\theta}{2}\right)$ and $\log \left(\sin \frac{\theta}{2}\right)$, Pacific J. Math., 168, 2 (1995), 271-289.

[15] X. WANG, Integral representations and binomial coefficients, J. Integer Sequences, 13 (2010), Article 10.6.4. 
[16] Feng-Zhen Zhao and Tianming Wang, Some results for sums of the inverse binomial coeffcients, Integers, 5 (2005), A22.

[17] I. J. ZuCKER, On the series $\sum_{k=1}^{\infty}\left(\begin{array}{c}2 k \\ k\end{array}\right)^{-1} k^{-n}$ and related sums, J. Number Theory, 20 (1985), 92-102. 